\title{
DISPOSITIF INTERFÉRENTIEL DESTINÉ \\ A LA MESURE DE L'ÉPAISSEUR MÉCANIQUE DE COUCHES MINCES
}

\author{
par H. DUPOISOT et P. LOSTIS, \\ Institut d'Optique, Paris.
}

Résumé. - Cet article montre comment il est possible d'inverser le contraste des franges à ondes multiples et ainsi d'améliorer le pointé de ces franges.

Cette méthode a été utilisée pour mesurer l'épaisseur mécanique de couches minces et contròler la planéité de support. La précision est de l'ordre de 3 Angströms.

Abstract. - The article shows bow it is possible to reverse the contrast of multiple wave fringes and consequently improve the sharpness of these fringes. This method has been utilized to measure the mechanical thickness of thin films and to control the flatness of the support. The precision is of the order of 3 Angströms.

Les propriétés physiques des couches minces sont liées à un certain nombre de données essentielles, parmi lesquelles figurent notamment la détermination de l'épaisseur et l'étude de la structure. Ces problèmes ont suscité l'élaboration et le développement de nombreuses techniques mécaniques, électriques et optiques ; parmi ces dernières, une méthode interférentielle proposée par S. Tolansky [1] a retenu spécialement notre attention. Après avoir apporté quelques améliorations à la méthode, nous avons été conduits à la réalisation d'un dispositif de grande précision et d'utilisation aisée et rapide.

L'appareil se compose principalement d'un microscope métallographique et d'un interféromètre Fizeau-Tolansky placé sur la platine d'observation. La figure 1 fournit quelques détails du montage optique réalisé.

L'éclairage est assuré par une source S émettant

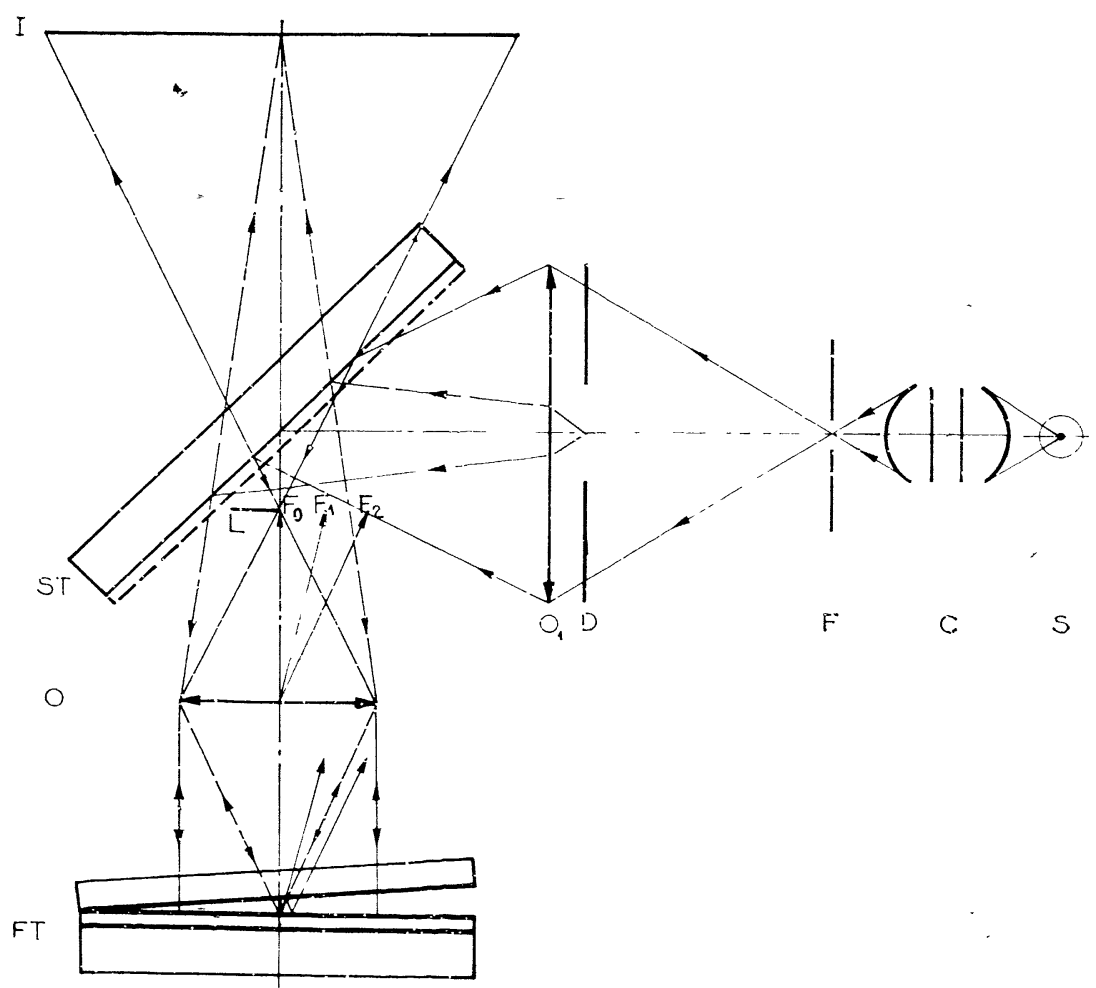

FIG. 1. 
un spectre visible et discret. A près convergence en F, puis réflexion sur une séparatrice semi-transparente $\mathrm{S}-\mathrm{T}$, les rayons lumineux sont condensés dans le plan focal de l'objectif $\mathrm{O}$ du microscope. L'interféromètre, constitué de deux miroirs fortement réfléchissants faisant entre eux un angle $\theta$, est ainsi éclairé par un faisceau de lumière parallèle, de faible ouverture, rendu éventuellement monochromatique. Le miroir inférieur de l'interféromètre est réalisé de la façon suivante : un support vitreux est recouvert sur la moitié de sa surface par la couche à étudier, puis l'ensemble est totalement métallisé par un dépôt opaque et uniforme.

Considérons, à présent, la répartition locale de l'intensité $I(\varphi)$, dans le plan image $I$.

Les franges, résultant de l'interaction des ondes issues de $F_{0}, F_{1} \ldots F_{n}$, points de convergence des faisceaux réfléchis, apparaissent sombres sur fond clair. Elles ont, au voisinage immédiat de l'image de l'arête du coin d'air, l'aspect de franges de FabryPerot, de type réflexion ( $f$ g. $2 a$ ). L'inversion de contraste peut être obtenue par déphasage et absorption totale (fig. 2b) ou partielle (fig. 2c) de

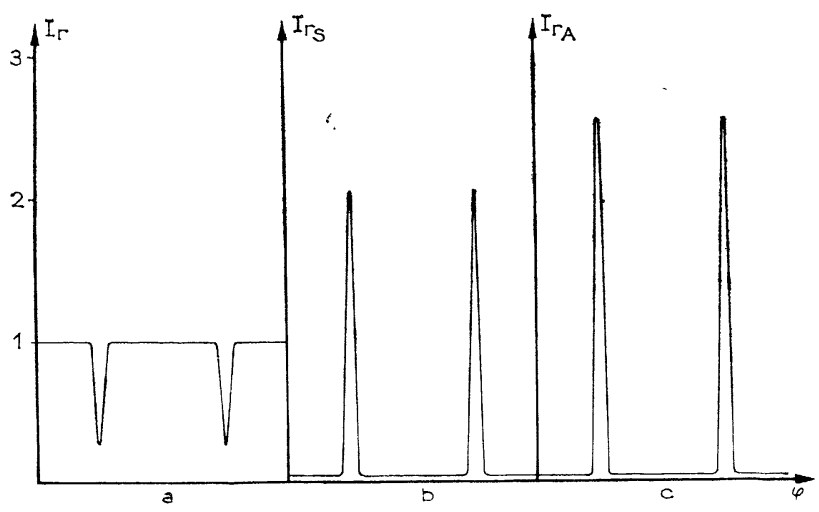

FIG. 2.

l'onde directe associée à $\mathrm{F}_{0}$ : la répartition de l'intensité est alors identique à celle d'une figure d'interférences par transmission. Les franges possèdent, en outre, l'avantage d'être extrêmement lumineuses et contrastées. Pour la longueur d'onde $\lambda=5461 \AA$, les facteurs de réflexion du miroir supérieur non absorbant et du miroir inférieur absorbant étaient respectivement 0,89 et 0,92 : dans ces conditions expérimentales, le contraste $C$, caractérisé par le quotient de la variation maximale de l'intensité, $I_{\max }-I_{\min }$, et de la valeur de son maximum $I_{\max }$, passe, au cours de l'inversion, de 0,734 à 0,997 . Cet accroissement est en fait d'autant plus important que les franges, sur lesquelles porte l'observa. tion, ont un ordre plus élevé.

La fonction représentant l'évolution du contraste de franges de Tolansky tend en effet vers zéro lorsque l'ordre $m$ augmente ( $f g .3$ ). Il existe une valeur $m$, correspondant au seuil de visibilité
$C_{1}=0,02$, au-delà de laquelle toute observation visuelle de franges devient impossible [2]. Cette limite est d'autant plus faible que l'angle $\theta$ de l'interféromètre est plus grand. A titre d'exemple, pour les deux miroirs précédemment définis et pour $\theta=0,002$ radian, le contraste $C_{1}$ de l'objet décroît de 0,734 pour $m=0$ à 0,433 pour $m=40$. Après inversion, les franges ont par contre une visibilité constante, indépendante de l'ordre, ce qui s'explique par le fait que les maximums et minimums successits de la fonction $I$ décroissent dans le même rapport lorsque $m$ augmente. Le quotient $\mathrm{AC} / \mathrm{AB}$ exprime l'amplification de contraste obtenue pour une valeur donnée de $m$.

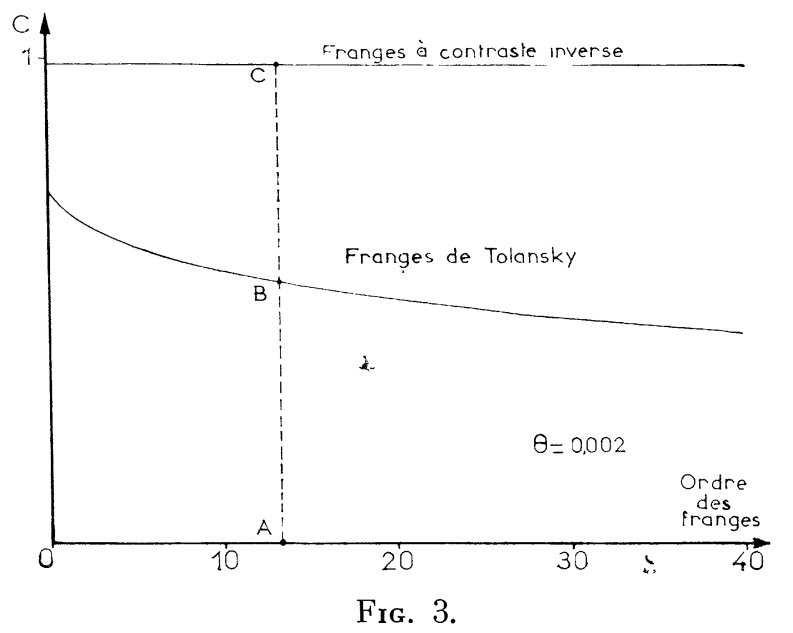

La figure 4 présente quelques éléments essentiels du dispositif, notamment l'interféromètre A, l'inverseur de contraste $B$.

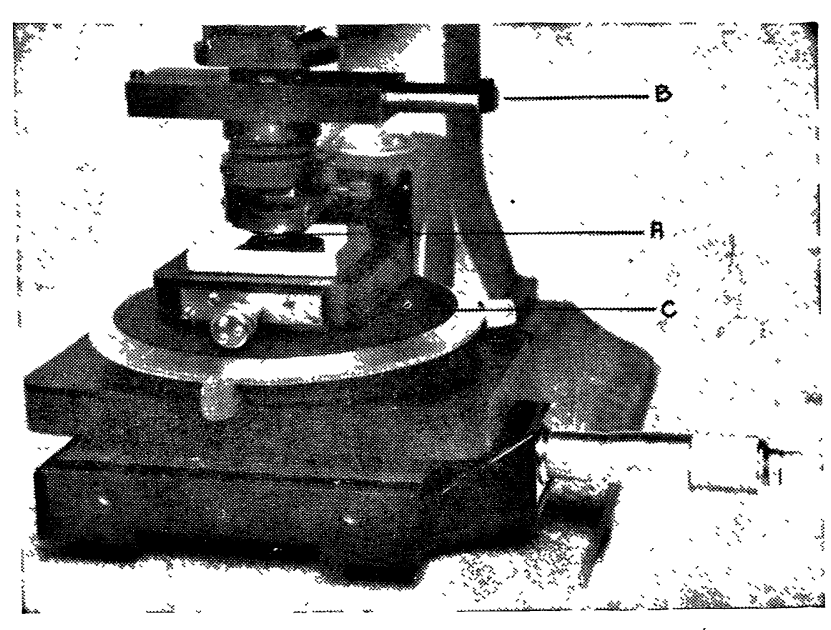

FIG. 4.

La plateforme C supportant le miroir inférieur possède trois degrés de liberté grâce auxquels la 
ligne de décalage des franges est amenée au centre du champ de vision, position où elle est maintenue lors de translations parallèles à la discontinuité. Le miroir supérieur est solidaire de l'un des côtés d'un triangle déformable adapté à l'objectif du microscope. La flexion d'une membrane métallique et la rotation de la monture du miroir autour d'une charnière élastique permettent de faire varier l'angle $\theta$ de 0 à 20 minutes et de choisir ainsi les fréquences spatiales constituant l'objet. L'utilisateur peut, entre autres possibilités, régler la périodicité de l'image observée visuellement à la valeur de $10000(\lambda / 2)$. Un micromètre oculaire, gradué au micron, permet alors, par simple observation, de connaître la valeur de la dénivellation exprimée en
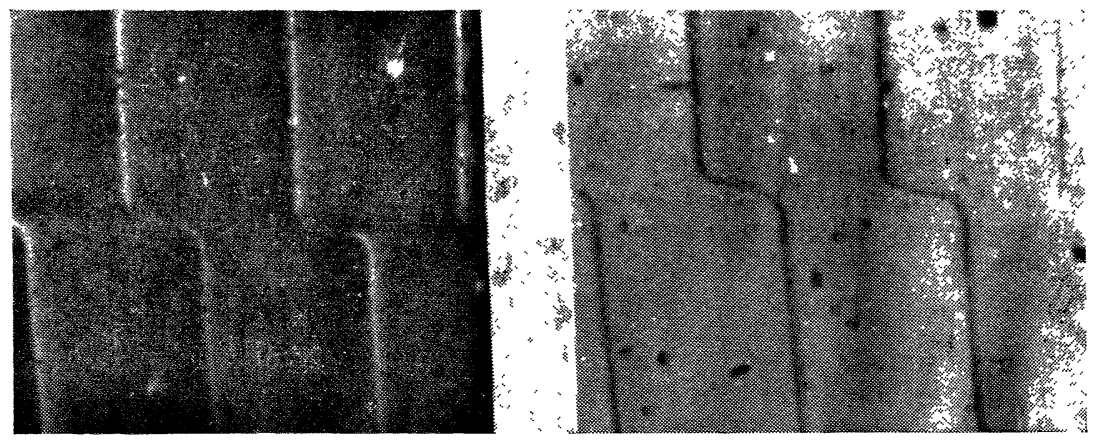

FIG. 5.

laire à la discontinuité, ce qui se traduit par un changement visible de la pente des franges. On peut également déterminer toute irrégularité de relief. On remarquera que les franges de la figure 5 ne présentent pas de décalage à angle droit.

Une dénivellation de type progressif, de réalisation aisée, simplifie les mesures, lorsque le décalage atteint plusieurs interfranges. Dans ce cas, il est même préférable d'utiliser plusieurs radiations : en effet, la disposition relative des franges produites par les différentes radiations permet de mesurer sans ambiguïté le nombre entier d'interfranges qui correspond à la discontinuité étudiée.
Angströms. On réalise ainsi un dispositif de lecture à interpolation directe.

Une inversion purement optique a également été réalisée, en collaboration avec MM. Françon et Prat. Lors de cette expérience, le support du miroir $M_{1}$ était constitué d'un polariscope de Savart, placé entre deux polariseurs convenablement orientés. Un certain nombre de foyers équidistants, dont le foyer principal $F_{0}$, sont ainsi susceptibles d'être supprimés. Il est même possible d'éliminer une onde réfléchie sur deux, ce qui pourrait être avantageux dans le cas de très faibles décalages [3].

La figure 5 est une photographie des franges. Elle met en évidence la possibilité de détecter facilement tout gradient d'épaisseur de direction perpendicu-

Quant à la détermination visuelle de l'excédent fractionnaire du dit décalage, l'expérience montre que la coïncidence de la référence mobile du micromètre et des franges s'obtient plus facilement lorsque le contraste est inversé.

On notera, enfin, que la même technique peut également servir à l'étude de la planéité d'une surface quelconque, préalablement métallisée. La précision que l'on peut espérer atteindre est de l'ordre de $\lambda / 500$.

Manuscrit reçu le 30 novembre 1965.

\section{BIBLIOGRAPHIE}

[1] Tolansky (S.), Multiple - beam interferometry of surfaces and films, Clarend on Press, Oxford, 1948.

[2] Legrand (Y.), Optique physiologique, Édition de la Revue d'Optique.
[3] Dupoisot (H.), Thèse, Paris, 26 juin 1965, à publier dans Revue d'Optique. 